\title{
EFFECT OF UNIAXIAL STRETCHING ON STRUCTURAL PROPERTIES OF POLY(3-ALKYLTHIOPHENES)
}

\author{
W. ŁUŻNY
}

Faculty of Physics and Nuclear Techniques, University of Mining and Metallurgy Al. Mickiewicza 30, 30-059 Cracow, Poland

AND M. ZAGóRSKA

Chemical Department, Warsaw Technical University

Noakowskiego 3, 00-664 Warszawa, Poland

(Received March 25, 1997)

The influence of uniaxial strctcling on the molecular structure of poly(3-alkylthiophenes) was studied with the use of X-ray diffraction. Three poly(3-alkylthiophenes), namely these containing $n$-hexyl; $n$-octyl and $n$-decyl groups, were investigated. The samples were oriented by stretching and the diffraction patterns for two geometries of scattering were obtained for undoped samples. The comparison of experimental difraction data for unoriented and oriented samples, gives information about the role of the side chains in the changes of molecular conformation induced by stretching of the polymer sample. The main conclusion of our work is that the process of orientation induces similar phenomena in all samples studied, but the structural and conformation changes depend on the side chain length: the shorter the alkyl substituent, the easier the straightening of the main polymer chain. The authors suggest that uniaxial stretching of poly(3-alkylthiophenes) changes the degree of main chain planarity. This effect can influence the $\pi$-conjugation in polymer system, which may have great importance for any future application of poly(3-alkylthiophenes).

PACS numbers: $36,20 .-\mathbf{r}, 61.10 .-\mathrm{i}$

\section{Introduction}

The structure of pure and doped poly(3-alkylthiophenes) (PATs) has been investigated by many research workers because of its importance for the electronic and other physical properties of this class of conjugated polymers (see for example [1-6]); especially, the works of Winokur and co-workers $[7,8]$ have thrown the light onto the structure properties of PATs. The problem of the side chains has been 
investigated also by Levon and co-workers [9] and by the group of Yoshino [10]. The main problem comnected with the interpretation of experimental diffraction data is the influence of the molecular conformation of the polymer system on the relative intensity of diffraction peaks. Ilowever, a considerable development in this field has also been achieved recently [11-13]. One can state that the basic features of the structure of PATs are well understood now; except for some less important details, the origin of all diffraction peaks is rather clear. The main goal of this work is to describe the influence of alkyl group length on the structure of unoriented and oriented samples of poly(3-alkylthiophenes). This is an interesting problem taking into account the importance of alkyl sulstituents in the preparation of soluble thus solution processable conducting polymers.

\section{Experimental}

The exact procedure for the polymerization of PATs was reported in [14]. In this work, three types of samples were investigated: poly(3-decylthiophene) (PDT), poly(3-octylthiophene) (POT) and poly(3-hexylthiophene) (PITT). The films were cast from $\mathrm{CIICl}$ solution at room temperature. The X-ray diffraction experiments were carried out on the IIZG-4 wide-angle X-ray diffractometer. A Cu-anode tube with a Ni-filter was used and a crystal monochromator was placed behind the sample. The measurements were performed in a typical Bragg geometry $(\theta, 2 \theta)$ in a reflection mode. Each sample was measured three times. The first diffractogram was obtained for the unstretched sample and the two other diffractograms were obtained for the stretched sample in two configurations: when the stretching direction is perpendicular to the plane of an incident beam and a reflected beam, and when the stretching direction is parallel to this plane. This type of experimental geometry is very often used for the structural investigations of oriented polymers and this technique is explained in [15]. In principle, it allows to obtain a measure of the average orientation of the polymer chains in the direction of the symmetry axis $Z$ (the direction of stretching). This measure can be expressed as

$$
f=\left(3\left\langle\cos ^{2} \sigma\right\rangle-1\right) / 2,
$$

where $f$ is the so-called orientation function and $\left\langle\cos ^{2} \sigma\right\rangle$ is the average square cosine of the angle $\sigma$ between the $Z$ axis and the chain axis of the polymer. However, in this case it is necessary to measure the full azimuthal scans of the diffraction peaks, not only their two values, as in our case. Therefore, we were able to obtain only qualitative information about the orientation of the polymer chains after stretching. The strength of an anisotropy of the diffraction reflections gives a measure of the orientation degree.

Possible "in plane" anisotropy of the unstretched samples was excluded by careful X-ray studies. In the cast film some texture effects are sometimes found in the direction perpendicular to the surface of the film. In our case it is rather difficult to verify it, taking into account small thickness of the film. However, isotropy within the film plane is of crucial importance for future comparison with stretched films. We tested this "in plane" isotropy by rotating the film around the axis perpendicular to the plane of the sample. The samples (films of the thickness 40 to $60 \mu \mathrm{m}$ ) were stretched with the use of the home-made stretching apparatus 
placed on the goniometer. Every sample was stretched 1.5 times of its original length. As it will be shown below, this relatively little alignment is quite sufficient to obtain a significantly oriented polymer sample. The degree of orientation is not very high. The samples were constrained during the $\mathrm{X}$-ray measurements and there were no significant differences in the volume of samples for the different configurations of the measurement. The drawing process was performed in the room temperature.

The experimental diffractograms were obtained by measuring the intensity of scattered radiation in discrete steps of $2 \theta=0.05^{\circ}$ and the number of counts for the highest peaks exceeded 5000 counts for one experimental point. This leads to ca. $3 \%$ statistical error for the smallest diffraction peaks. This statistics was quite satisfactory since the orientation induced changes were statistically meaningful. Because the numerical smoothing procedure was applied to the diffraction data, the noise was significantly reduced and all features of the diffraction patterns referred to in the text can be considered despite their very low (in some cases) relative intensity.

\section{Results of the computer modelling}

Because of the small number of the difraction peaks, the interpretation of the polymer diffraction data is possible only when the experiment is supported by the computer modelling. In the case of PATs, such work has been done and it is described in detail in [13]. Some conclusions have been also presented in [12]. Therefore, in this article, we only present the basic steps of the procedure and its most interesting and needed here results. The idea is simple: one can assume the crystal structure and conformation, then one computes the diffraction pattern for such structure and finally, one compares the results of modelling with the experimental difractogram. This procedure should be repeated until the correlation is satisfactory. Of course, this procedure gives only positions, intensities and indexations of the diffraction peaks, without background and without broadening of the reflections. The starting point of the modelling is the flat system of four thiophene rings with four decyl groups (see Fig. 1). The main conclusions are as follows:
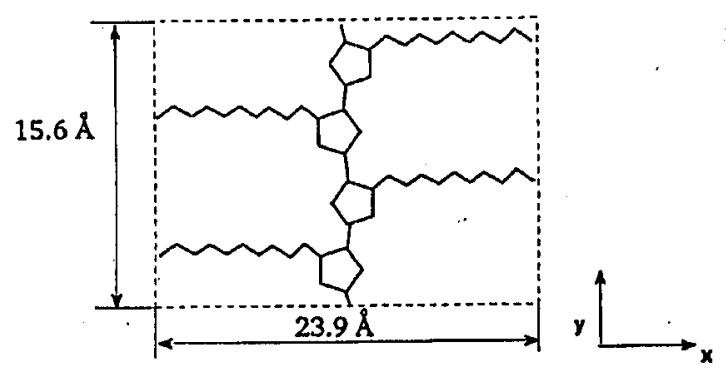

Fig. 1. Scheme of the model structure of PDT within one unit cell. 

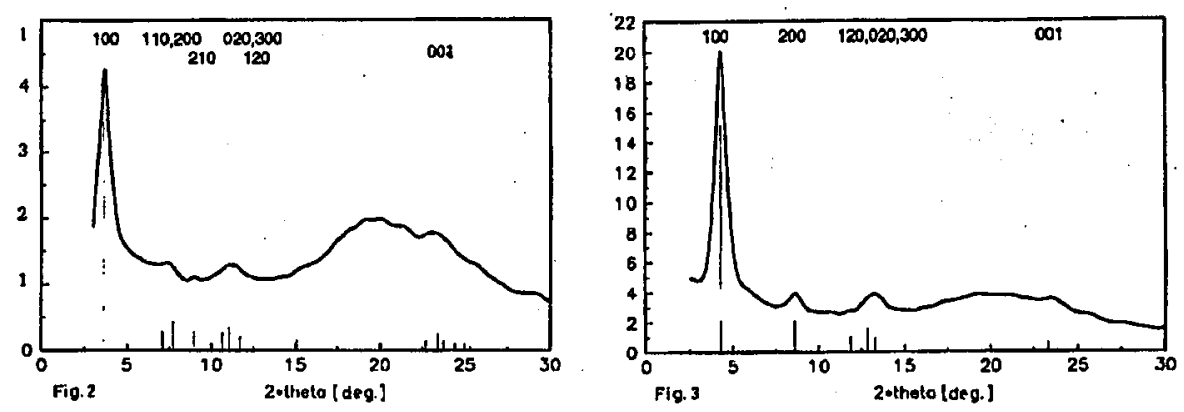

Fig. 2. X-ray diffraction pattern for unoriented sample of poly(3-decylthiophene). The vertical lines give the positions and intensitics of the calculated diffraction peaks. The numbers give the indexation of these peaks, $I-$ intensity in arbitrary units.

Fig. 3. X-ray diffraction pattern for unoriented sample of poly(3-octylthiophene); the description as in Fig. 2.

the greater are the torsion angles, the smaller become the ratios of the intensities of three diffraction reflections to the main peak $\left(\begin{array}{lll}1 & 0 & 0\end{array}\right)$. The best agreement with experimental diffraction data has been obtained for the average torsion angle in the range $45^{\circ}$ to $50^{\circ}$.

The intensity of the reflection $\left(\begin{array}{lll}0 & 0 & 1\end{array}\right)$, related to the repetition distance between the polymer chains in the direction perpendicular to their planes, quickly decreases for the torsion angles increasing. All details of the model assumed, together with the discussion and the results, are explained in [13].

The results of the computer modelling for the diffraction by PATs, together with the appropriate experimental diffraction pattern, are presented in Figs. 2 to 4. The vertical lines give the calculated positions and intensities of the diffraction peaks, and the numbers in the upper part of the diagrams give the indexations of these peaks. One can conclude from these results that the assumed model of the structure of PATs explains the basic features of the experimental diffraction data quite well. More exact analysis of the obtained results is presented in the next section, where we make use of the fact that the results of the computer modelling allow us to explain the origin of the diffraction peaks and, moreover, to explain the influence of stretching on the experimental diffraction patterns.

There is no possibility to obtain direct, quantitative information on the conformation of oriented polymers from the computer modelling, because the stretched samples cannot be treated as an ideal powder; they exhibit strong texture. Therefore, there is no way to find an exact correlation between the relative intensity of the diffraction peaks and the parameters of conformation of the stretched polymer sample. One can only get some qualitative information from the change of diffraction pattern obtained after orientation.

One more point should be underlined here: as implies from the computer modelling, the change of the conformation parameters (related to the change of the atom positions within the unit cell) induces the change in the intensity of the diffraction peaks. Therefore, one cannot directly compare the intensity of any 


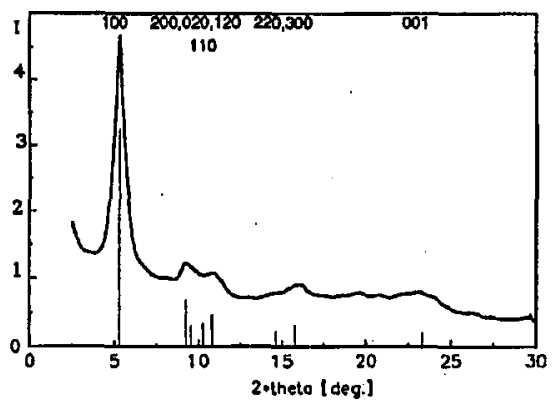

Fig. 4. X-ray diffraction pattern for unoriented sample of poly(3-hexylthiophene); the description as in Fig. 2.

diffraction peak (for instance, $\left(\begin{array}{lll}1 & 0 & 0\end{array}\right)$ ) for the unstretched and stretched sample. Simply, the total scattering intensity (assuming an angular integration) may not be constant after ordering of the sample, if one assumes stretch induced changes in the unit cell structure.

\section{Experimental results and discussion}

Diffraction patterns of the unoriented samples are collected in Fig. 5. All diffractograms have the crystalline component as well as the amorphous one which is rather typical of almost all conjugated polymers. The discussion of the amorphous component of the PATs diffraction data has been presented elsewhere [16]; here, we will concentrate on the characteristic features of the crystalline component of all diffractograms obtained.

In further discussion we assume an orthorhombic unit cell with the following set of the lattice constants: $a=23.9, b=7.8$ and $c=3.8 \AA$ [13].

The diffraction pattern obtained for unoriented PDT is now better understood. The main peak at $2 \theta=3.70^{\circ}$ corresponds to the interplanar distance $d=23.9 \AA$; it originates from the distance between the main chains of the polymer

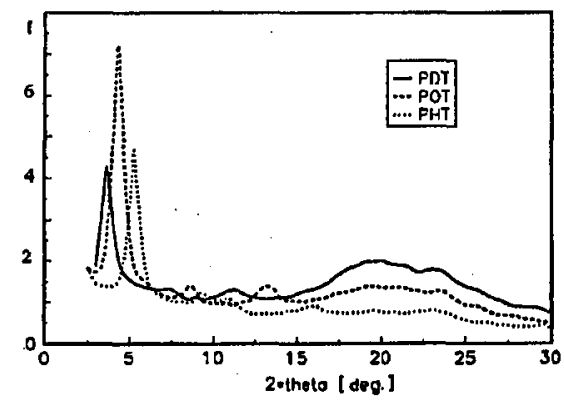

Fig. 5. X-ray diffraction patterns for unoriented samples of poly(3-alkylthiophenes): poly(3-decylthiophene) (PDT), poly(3-octylthiophene) (POT) and poly(3-hexylthiophene) (PHT). 
in their planes and usually it is marked as a reflection $\left(\begin{array}{lll}1 & 0 & 0\end{array}\right)$. The second peak, for $20=7.30^{\circ}$ is the sum of two reflections: $(200)$ and $\left(\begin{array}{lll}1 & 1 & 0\end{array}\right)$ (see Fig. 2). There is a little, but distinct peak for $20=8.95^{\circ}$; as implies from Fig. 2, it should be indexed

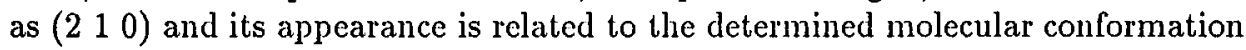
of the polymer. As results from the intensity calculations in computer modelling, this peak can appear for the system with non-planar main chain (for example, with the average torsion angle between two adjacent thiophene rings contained in the range $40^{\circ}$ to $50^{\circ}$ ) and when the majority of the side chains are perpendicular to the axis of the main chain. The next, broader maximum for $2 \theta=11.20^{\circ}$ and $d=7.8 \AA$ has the intensity as high as the reflection $(200)$ and therefore it cannot be explained by indexes ( $\left.\begin{array}{lll}3 & 0 & 0\end{array}\right)$ only (as is usually being done in the literature); indeed, our results of the computer modelling have shown that this maximum is the sum of three reflections: $\left(\begin{array}{lll}3 & 0 & 0\end{array}\right),\left(\begin{array}{lll}0 & 2 & 0\end{array}\right)$ and $\left(\begin{array}{lll}1 & 2 & 0\end{array}\right)$. This second component is related to the repetition distance of the two thiophene rings along the main chain. At last, the maximum for $20=23.10^{\circ}$ and $d=3.9 \AA$ can be described as the reflection $\left(\begin{array}{lll}0 & 0 & 1\end{array}\right)$ and it originates from the distance between the planes of the polymer cliains.

The comparison of the diffractograms obtained for unoriented PDT, POT and PIIT i.e. for samples with decreasing side chain length leads us to two conclusions. First of all, the main crystalline peak with indexes $\left(\begin{array}{lll}1 & 0 & 0\end{array}\right)$ is shifted towards shorter distances as the length of the side chain decreases: its position is $20=3.70^{\circ}(d=23.9 \AA), 20=4.30^{\circ}(d=20.5 \AA)$ and $20=5.30^{\circ}(d=16.7 \AA)$ for PDT, POT and PIIT respectively. This effect is connected with the possibility of closer stacking of the polymer chains in their planes for the shorter side chains and it was reported in [1]. Second, the small peak for $20=23.10^{\circ}$ is present in all difractograms and this observation is clearly understood; the distance between the planes of the polymer chains should not depend on the length of the side groups.

The experimental diffractograms obtained for oriented PDT are presented in Fig. 6. The main feature of these patterns is an anisotropy of the difraction peaks, seen both for crystalline and amorphous component of scattered intensity; the second, very distinct effect consists of the enhancement of the relative intensity of the third diffraction peak. If one takes into account the results of the computer modelling, it may be concluded that the average value of the torsion angle between two adjacent thiophene rings has lowered considerably after stretching. Strictly speaking, the elongation of the polymer sample implies straightening of the polymer chains; they become more flat than for unoriented sample. This conclusion is supported by the lack of the reflection $\left(\begin{array}{lll}2 & 1 & 0\end{array}\right)$. The anisotropy of the polymer chains induced in pure PDT system for these low elongation ratios is rather weak.

Three diffractograms obtained for unoriented and oriented POT are presented in Fig. 7. The origin of the peaks visible in this cliagram is clear if one takes into account the conclusions from Fig. 3. The first two peaks have indexes $\left(\begin{array}{lll}1 & 0 & 0\end{array}\right)$ and $\left(\begin{array}{lll}2 & 0 & 0\end{array}\right)$. The third one is the sum of reflections with indexes: $\left(\begin{array}{lll}0 & 2 & 0\end{array}\right),\left(\begin{array}{lll}1 & 2 & 0\end{array}\right)$ and $\left(\begin{array}{lll}3 & 0 & 0\end{array}\right)$.

The changes in experimental diffractograms induced by stretching of POT sample can be summarized as follows. There is a distinct anisotropy of the first peak $\left(\begin{array}{lll}1 & 0 & 0\end{array}\right)$, connected with the orienting of the main chains. The increase in 

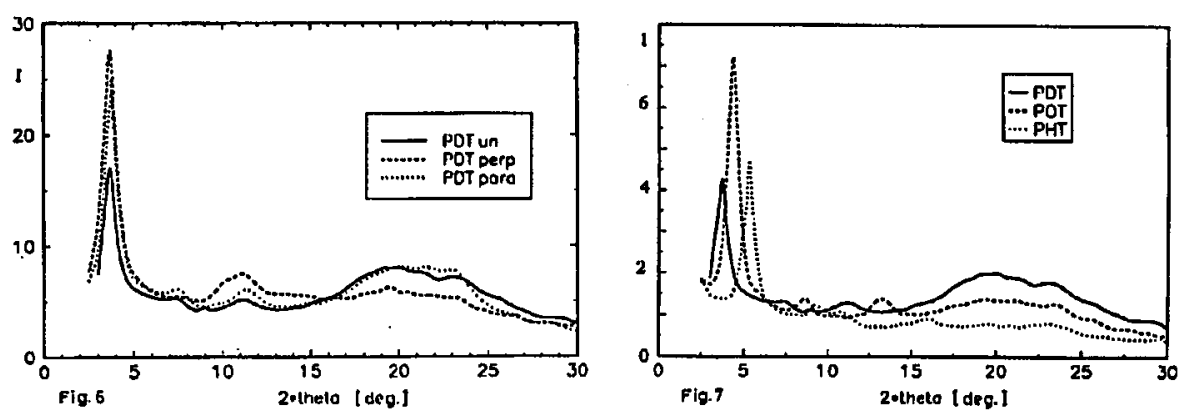

Fig. 6. X-ray diftraction patterns for unoriented PDT and oriented PDT perpendicular and parallel to the sample stretching direction.

Fig. 7. X-ray diffraction patterns for unoriented POT and oriented POT perpendicular and parallel to the sample stretching direction.

intensity of the third maximum for the diffraction pattern obtained in the perpendicular direction, is certainly induced by straightening of the main chains and lowering of the average torsion angle for two adjacent thiophene rings. Ilowever, the enhancement of the relative intensity for the third diffraction peak is perhaps not so obvious. Also, contrary to the case of PDT, there is no anisotropy of the amorphous component of diffiaction patterns.

In Fig. 8 the experimculal diffractograms obtained for unoriented and oriented PIIT are collected. The first maximum has indexes $\left(\begin{array}{lll}1 & 0 & 0\end{array}\right)$ and its behaviour after stretching gives the evidence for the anisotropy of the main chains appearing in the polymer system after orienting. The second peak is now splitted into two small peaks. Following the results of computer modelling it can be explained by three sets of indexes: $\left(\begin{array}{lll}2 & 0 & 0\end{array}\right),\left(\begin{array}{lll}0 & 2 & 0\end{array}\right)$ and $\left(\begin{array}{lll}1 & 2 & 0\end{array}\right)$. The third, a very weak maximum (for $\left.20=16.30^{\circ}\right)$ is the sum of two reflections: $\left(\begin{array}{lll}3 & 0 & 0\end{array}\right)$ and $\left(\begin{array}{lll}2 & 2 & 0\end{array}\right)$.

The changes induced in this case by stretching are very remarkable, especially for the diffraction data obtained for the perpendicular direction. Now, the enhancement of intensity of the maximum for $20=11.30^{\circ}$ (connected with the order along the main chain) is really large. This observation suggests that the conformation changes induced in the polymer system by stretching are particularly facilitated in this case, e.g. for short side cliains.

The explanation of the differences observed for the samples with various lengths of the side chains is well confirmed by joint presentation of the diffraction data obtained in perpendicular direction for all three samples studied, as it is shown in Fig. 9. One can say that the common feature of three patterns presented in this picture is the enhancement of intensity for the maximum at $2 \theta=11.30^{\circ}$. As it has already been explained above, this is connected with the lowering of the average torsion angle between the adjacent thiophene rings and with the straightening of the main polymer chain.

As has been mentioned above, the strength of the anisotropy of the diffraction peaks can give some information on the degree of orientation. If we define $I_{\text {perp }}^{100}$, $I_{\text {para }}^{100}$ and $I_{\text {un }}^{100}$ as an integrated intensily (after subtraction of the background) of 

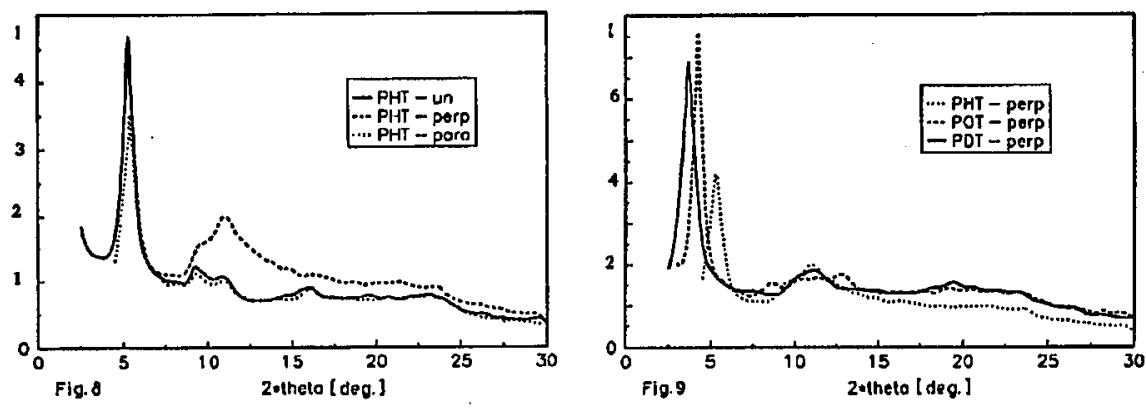

Fig. 8. X-ray diffraction patterns for unoriented PIIT and oriented PHT perpendicular and parallel to the sample stretching direction.

Fig. 9. X-ray difraction patterns for oriented samples of PATs. The direction of the stretching is perpendicular to the plane of two beams: an incident one and a reflected one.

the peak $\left(\begin{array}{lll}1 & 0 & 0\end{array}\right)$ for perpendicular diffraction scan, parallel one, and unorienting sample diffraction, respectively, then it is possible to calculate the parameter of anisotropy as the ratio of $I_{\text {perp }}^{100}$ to $I_{\text {para }}^{100}$. For the three polymer samples studied, the value of this ratio is within 1.2 to 1.5 .

It is not easy to explain this observation. This can be done here if one compares the values of $I^{100}$ for three polymer samples investigated, it turns out that

$$
\begin{array}{ll}
I_{\text {un }}^{100}<I_{\text {para }}^{100}<I_{\text {perp }}^{100} & \text { for PDT, } \\
I_{\text {para }}^{100}<I_{\text {un }}^{100}<I_{\text {perp }}^{100} & \text { for POT, and } \\
I_{\text {para }}^{100}<I_{\text {perp }}^{100}<I_{\text {un }}^{100} & \text { for PIIT. }
\end{array}
$$

It seems that the anisotropy of the diffraction patterns is rather similar for all cases (vide supra). Different evolution of the diffraction patterns, caused by the orientation (for the same draw ratio) may be related to the different structure factor changes. This effect should be carefully investigated in the future.

One more point should be underlined at the end of this discussion. It is well known that there is a possibility of evaluating the average size $L$ of the crystalline domain from the width of the Bragg reflection, using the Scherrer formula

$$
L=\frac{K \lambda}{\Delta(2 \theta) \cos \theta},
$$

where $K$ is the constant close to 1 and $\Delta(20)$ is the full width at half-maximum of the reflection. Using this formula for the main peak ( $\left.\begin{array}{lll}1 & 0 & 0\end{array}\right)$ of the diffraction patterns studied, one can estimate the mean size of crystallites in the direction perpendicular to the axis of the main cliains and parallel to the plane of the main chains. For all unoriented samples studied, the value of $L$ ranges from 140 to $160 \AA$ and this value does not change after orienting the sample. This kind of behaviour suggests that orienting of undoped PATs consists mainly on the rearrangement of whole polymer crystalline region and only to a low degree it is connected with a change in the orientation of the polymer chains. 


\section{Conclusions}

The changes induced by stretching in the structure of PATs have been investigated with the use of X-ray diffraction. The influence of the various lengths of the side chain is now more clear: the shorter are alkyl groups, the easier it induces straightening the main polymer chain via drawing. This is an important information since the potential applications of conductive polymers are possible only for well ordered and well oriented systems. However, the length of the side chain has also significance for the polymerization process of PATs, namely for the percentage of couplings "head to head" and "head to tail", and this factor should be considered in further investigations.

Certainly, some comments should be done concerning the relation between our model of PATs structure and conformation, and other models existing in the literature $[1,4,11]$. We think that each model has its own advantages and its own weak points. In the case of PATs, where the samples have low or very low crystallinity, there is simply no possibility to get one model of the structure, which would be able to explain all structural and conformational properties of the polymer system. If the diffraction pattern consists of only few rather week and/or broad crystalline reflections, mixed with the amorphous component of the scattered intensity, thus one should not expect that it is possible to get one, unique and universal model of this polymer structure.

It has to be noticed that, by several authors (see, for example [4]), the macroscopic properties of the polymer film strongly depend on the "quality" of the sample. It seems reasonably to believe that the crystallinity, the maximum stretch ratio, the molecular weight, the polydispersity, and other properties may depend on many factors, which one cannot fully control, and which may strongly influence the structural properties of polymer sample, causing an important difference between various samples of "the same" polymer. It is worth adding that the stretch induced structural changes in doped PATs are much more distinct than in neutral samples [17].

\section{Acknowledgments}

We are indebted to Professor A. Pron and Professor S. Niziol for their help in the sample preparation and for the fruitful discussions. This work was financially supported by the grant of the Committee for Scientific Research No. 3 P405 05707.

\section{References}

[1] G. Gustaffson, O. Inganäs, O. Osterholm, J. Laakso, Polymer 32, 1575 (1991).

[2] J. Mårdalen, E.J. Samuelsen, O.R. Gautun, P.H. Carlsen, Solid State Commun. 77, 337 (1991).

[3] J. Mårdaleı, E.J. Samuelsen, O.R. Gautun, P.H. Carlsen, Solid State Commun. 80, 687 (1991).

[4] J. Mårdalen, E.J. Samuelsen, O.R. Gautun, P.II. Carlsen, Synth. Met. 48, 363 (1992).

[5] Y.F. Nicolau, P. Moser, J. Polymer Sci., Polymer Phys. 31, 1529 (1993).

[6] Proc. Int. Conf. on Synth. Metals, Göteborg 1992; Synth. Met. 55-57 (1993). 
[7] M.J. Winokur, P. Wamsley, J. Moulton, P. Snilh, A.J. Ileeger, Macromolecules 24, 3812 (1991).

[8] T.J. Prosa, M.J. Winokur, J. Moulton, P. Smith, A.J. Ileeger, Synth. Met. 55-57, 370 (1993).

[9] Wen-Ping IIsu, K. Levon, Ko-Shan Ilo, A.S. Myerson, T.K. Kwei, Macromolecules 26, 1318 (1993).

[10] K. Tashiro, Y. Minagawa, M. Kobayashi, S. Morita, T. Kawai, K. Yoshino, Synth. Met. 55-57, 321 (1993).

[11] T.J. Prosa, M.J. Winokur, J. Moulton, P. Smith, A.J. Ileeger, Macromolecules 25, 4364 (1992).

[12] W. Eużny, S. Nizioł, M. Zagórska, A. Proń, Synth. Met. 55-57, 359 (1993).

[13] W. Eużny, Acla Crystallogr. B 51, 255 (1995).

[14] I. Kulszewicz-Bajer, A: Pawlicka, J. Plenkiewicz, A. Pron', Synth. Met. 30, 61 (1989).

[15] I.M. Ward, Structure and Properties of Oriented Polymers, Applied Science, London 1975.

[16] W. Lużny, S. Nizioł, M. Zagórska, Synth. Met. 64, 59 (1994).

[17] W. Eużny, S. Niziol, G. Strączyński, A. Proń, Synlh. Met. 62, 273 (1994). 